\title{
Dock3 protects myelin in the cuprizone model for demyelination
}

\author{
K Namekata ${ }^{1}$, A Kimura ${ }^{1}$, C Harada ${ }^{1}$, Y Yoshida $^{2}$, Y Matsumoto ${ }^{1}$ and T Harada*,1,2
}

Dedicator of cytokinesis 3 (Dock3) belongs to an atypical family of the guanine nucleotide exchange factors. It is predominantly expressed in the neural tissues and causes cellular morphological changes by activating the small GTPase Rac1. We previously reported that Dock3 overexpression protects retinal ganglion cells from excitotoxic cell death. Oligodendrocytes are the myelinating cells of axons in the central nervous system and these cells are damaged in demyelinating disorders including multiple sclerosis (MS) and optic neuritis. In this study, we examined if Dock3 is expressed in oligodendrocytes and if increasing Dock3 signals can suppress demyelination in a cuprizone-induced demyelination model, an animal model of MS. We demonstrate that Dock3 is expressed in oligodendrocytes and Dock3 overexpression protects myelin in the corpus callosum following cuprizone treatment. Furthermore, we show that cuprizone demyelinates optic nerves and the extent of demyelination is ameliorated in mice overexpressing Dock3. Cuprizone treatment impairs visual function, which was demonstrated by multifocal electroretinograms, an established non-invasive method, and Dock3 overexpression prevented this effect. In mice overexpressing Dock3, Erk activation is increased, suggesting this may at least partly explain the observed protective effects. Our findings suggest that Dock3 may be a therapeutic target for demyelinating disorders including optic neuritis.

Cell Death and Disease (2014) 5, e1395; doi:10.1038/cddis.2014.357; published online 28 August 2014

Dedicator of cytokinesis 3 (Dock3), an atypical member of the guanine nucleotide exchange factors (GEFs), is predominantly expressed in the neural tissues and causes cellular morphological changes by activating the small GTPase Rac1. ${ }^{1-3}$ We previously reported that Dock3 is primarily expressed in retinal ganglion cells (RGCs) in the retina, and Dock3 overexpression protects RGCs from glutamate neurotoxicity and oxidative stress in a mouse model of normal tension glaucoma. ${ }^{4}$ Dock3 also stimulates axonal regeneration after optic nerve injury through several molecular mechanisms. ${ }^{5,6}$ Dock3 was initially identified as a binding protein of presenilin1, a major causative gene of early-onset familial Alzheimer's disease. ${ }^{7,8}$ Recent studies suggested a possibility that there is a causal relationship between Alzheimer's disease and glaucoma. ${ }^{9,10}$ In addition, a pericentric inversion breakpoint in the DOCK3 gene has been described in patients with attention-deficit hyperactivity disorder. ${ }^{11}$ These findings suggest an involvement of Dock3 in various neurological disorders. ${ }^{1}$

Multiple sclerosis (MS) is an inflammatory disease of the central nervous system (CNS) characterized by progressive immune-mediated destruction of the myelin sheath. One important complication in MS is optic neuritis. Since it can cause severe visual loss that is currently irreversible, especially in the optic-spinal form of MS or neuromyelitis optica, ${ }^{12,13}$ it draws much attention to finding a treatment that will restore the visual function. Oligodendrocytes, the myelinating cells of CNS axons, are highly vulnerable to excitotoxic signals mediated by glutamate receptors. ${ }^{14}$ Furthermore, demyelinating lesions caused by excitotoxins can be similar to those observed in MS. ${ }^{15}$ These observations indicate that oligodendrocyte excitotoxicity could be involved in the pathogenesis of demyelinating disorders. ${ }^{16,17}$ We recently reported that Dock3 protects RGCs from glutamate excitotoxicity and oxidative stress. ${ }^{4}$ These results suggest a possibility that Dock3 may protect oligodendrocytes in demyelinating disorders, but the expression or functions of Dock3 in oligodendrocytes are unknown.

Feeding of cuprizone (bis(cyclohexanone)-oxaldihydrazone) to mice induces a consistent, synchronous and anatomically reproducible demyelination in the corpus callosum. ${ }^{18-21}$ The specific susceptibility of oligodendrocytes has been attributed to the high metabolic demand of these glial cells required to maintain a vast expanse of myelin and the resulting vulnerability to a disturbed energy metabolism. In the present study, we demonstrated that Dock3 is expressed in oligodendrocytes and Dock3 overexpression protects myelin in the corpus callosum in the cuprizone-induced demyelination model, an animal model of MS. Furthermore, we show that cuprizone demyelinates the optic nerves and the extent of demyelination is ameliorated in mice overexpressing Dock3. Cuprizone treatment impairs visual function, which was

\footnotetext{
${ }^{1}$ Visual Research Project, Tokyo Metropolitan Institute of Medical Science, Tokyo, Japan and ${ }^{2}$ Department of Neuro-ophthalmology, Tokyo Metropolitan Neurological Hospital, Tokyo, Japan

*Corresponding author: T Harada, Visual Research Project, Tokyo Metropolitan Institute of Medical Science, 2-1-6 Kamikitazawa, Tokyo 156-8506, Japan. Tel: +81 36834 2338; Fax: +81 36834 2339; E-mail: harada-tk@igakuken.or.jp

Abbreviations: ASK1, apoptosis signal-regulating kinase 1; BDNF, brain-derived neurotrophic factor; CNPase, 2', $3^{\prime}$-cyclic nucleotide $3^{\prime}$-phosphodiesterase; CNS, central nervous system; Dock3, dedicator of cytokinesis 3; EAE, experimental autoimmune encephalomyelitis; GEF, guanine nucleotide exchange factor; MAPK, mitogen-activated protein kinase; MBP, myelin basic protein; mfERG, multifocal electroretinogram; MS, multiple sclerosis; NMDA, N-methyl-D-aspartate; RGC, retinal ganglion cell; RRMS, relapsing-remitting multiple sclerosis

Received 20.5.14; revised 11.7.14; accepted 21.7.14; Edited by A Verkhratsky
} 
demonstrated by multifocal electroretinograms (mfERGs), an established non-invasive method, and Dock3 overexpression prevented this effect. Our findings suggest that Dock3 may be a therapeutic target for demyelinating disorders including optic neuritis.

\section{Results}

Expression of Dock3 in oligodendrocytes. Dock3 is mainly localized to the CNS. ${ }^{1-3}$ To determine the cell types that express Dock3, we prepared culture cells of neurons, astrocytes and oligodendrocytes from adult mouse brain. Immunoblot analysis revealed that Dock3 is strongly expressed in Neu-N-positive hippocampal neurons, but not in glial fibrillary acidic protein (GFAP)-positive astrocytes (Figure 1a). Interestingly, we found that Dock3 is also expressed in NG2-positive oligodendrocytes. We determined that Dock3 is double-labeled with myelin basic protein (MBP) in cultured oligodendrocytes (Figure 1b). In addition, Dock3 was localized to CC-1-positive oligodendrocytes in optic nerves (Figure 1c). These results indicate that Dock3 is expressed in oligodendrocytes as well as in neurons in vivo.
Effects of Dock3 on myelin protection in the corpus callosum. Chronic treatment of cuprizone is known to induce demyelination in the corpus callosum. ${ }^{18,19}$ To explore the effects of Dock3 in oligodendrocytes, we prepared a cuprizone-induced demyelinating model using wild-type (WT) and Dock3 overexpression (Tg) mice. After 6 weeks of cuprizone treatment, demyelination was obvious in the corpus callosum of the WT mice, which was determined by luxol fast blue (LFB) and MBP staining (Figures 2a and b). However, the extent of demyelination was clearly less in Dock3 Tg mice (Figures 2a-c). We also performed immunoblot analysis to determine whether Dock3 overexpression affects the expression of $2^{\prime}, 3^{\prime}$-cyclic nucleotide $3^{\prime}$-phosphodiesterase (CNPase), another myelin protein, and Neu-N in the whole brain during cuprizone treatment (Figure 2d). In cuprizone-treated WT mice, CNPase expression was clearly reduced whereas Neu-N expression was unchanged, suggesting cuprizone treatment affects myelin but not neurons. In Dock3 Tg mice, cuprizone-induced CNPase reduction was significantly suppressed. These results suggest that Dock3 protects oligodendrocytes during chronic demyelination. a

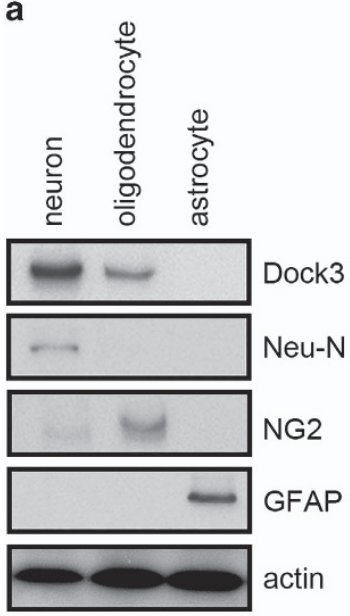

C
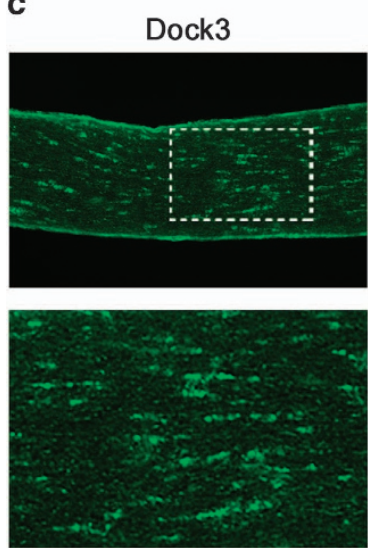

b
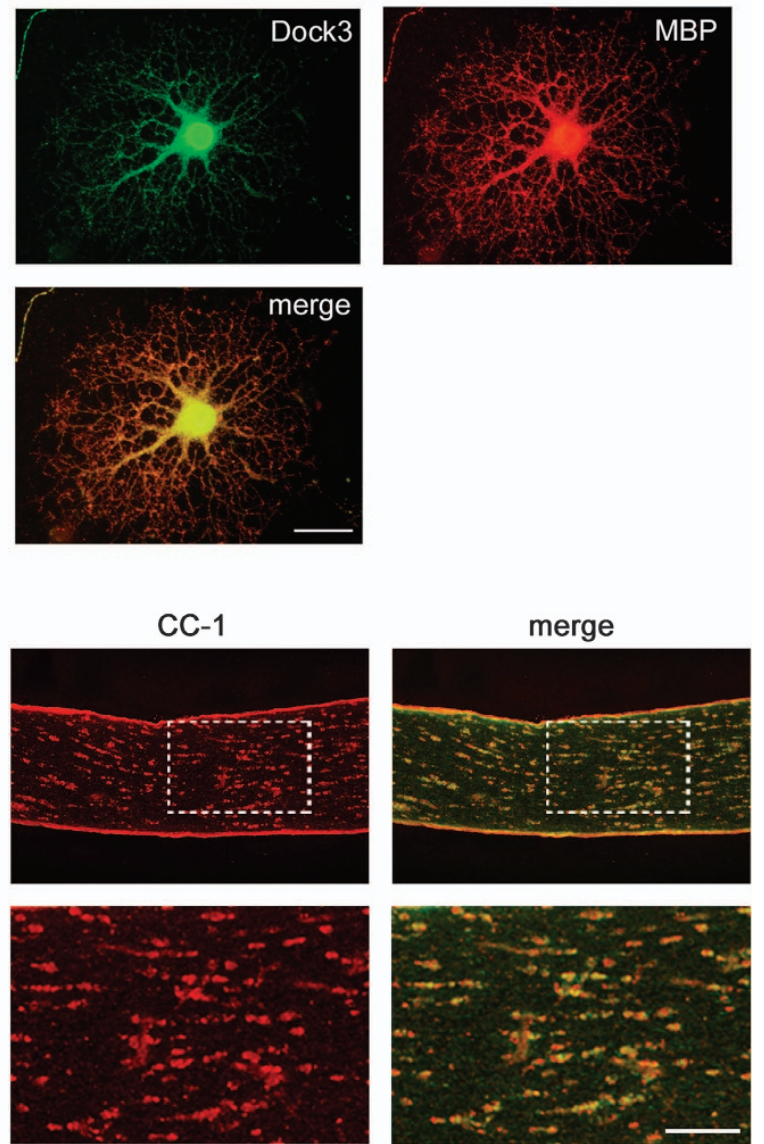

Figure 1 Expression of Dock3 in oligodendrocytes. (a) Expression of Dock3 and markers for neurons (Neu-N), oligodendrocytes (NG2) and astrocytes (GFAP) in cultured cells prepared from mouse brain. Note the expression of Dock3 in oligodendrocytes as well as in neurons. (b and $\mathbf{c}$ ) Double-labeling immunohistochemistry for Dock3 and myelin markers in cultured oligodendrocyte (b) and optic nerve (c). Scale bar: $20 \mu \mathrm{m}$ in b and 100 and $50 \mu \mathrm{m}$ in the upper and lower panels in $\mathbf{C}$ 
a

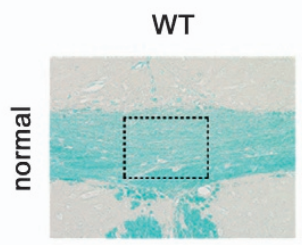

LFB
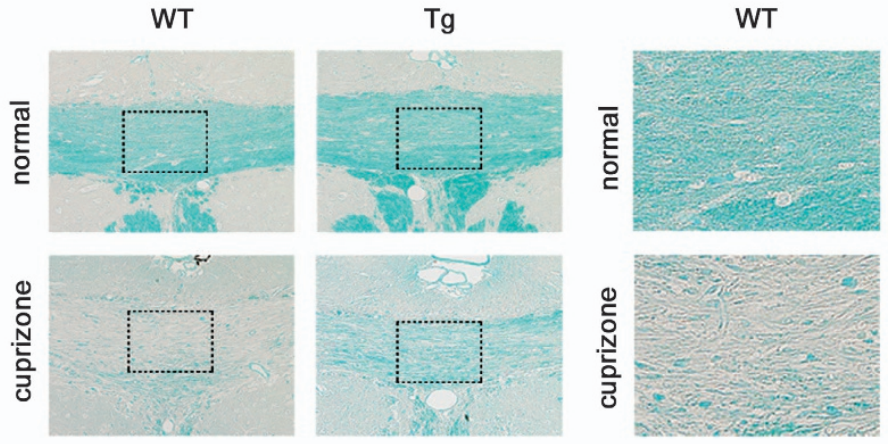

$\mathrm{Tg}$
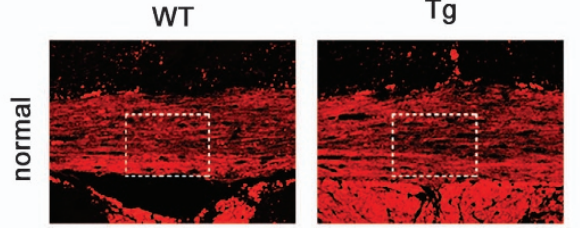

MBP
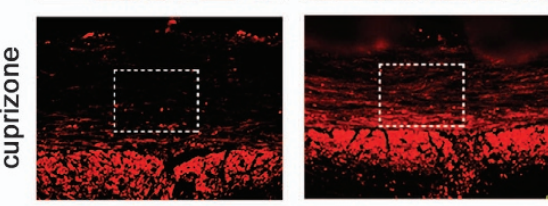

$\mathrm{Tg}$
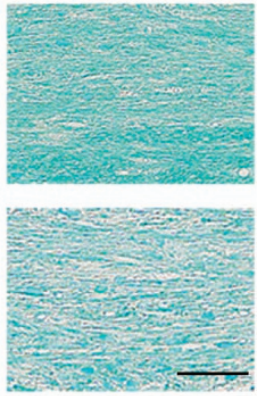

WT
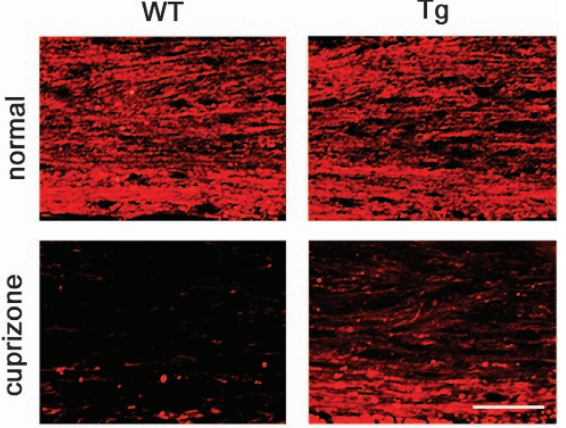

C
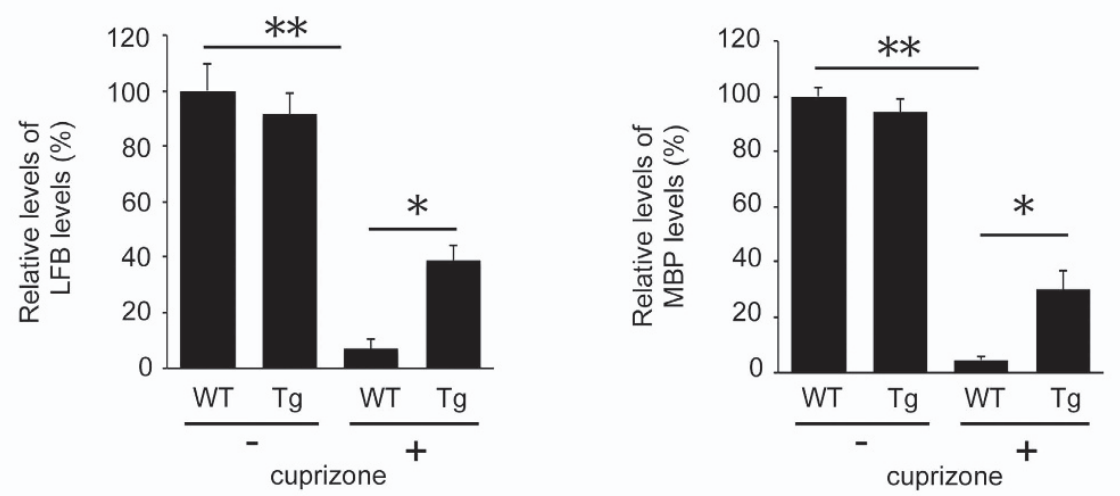

d
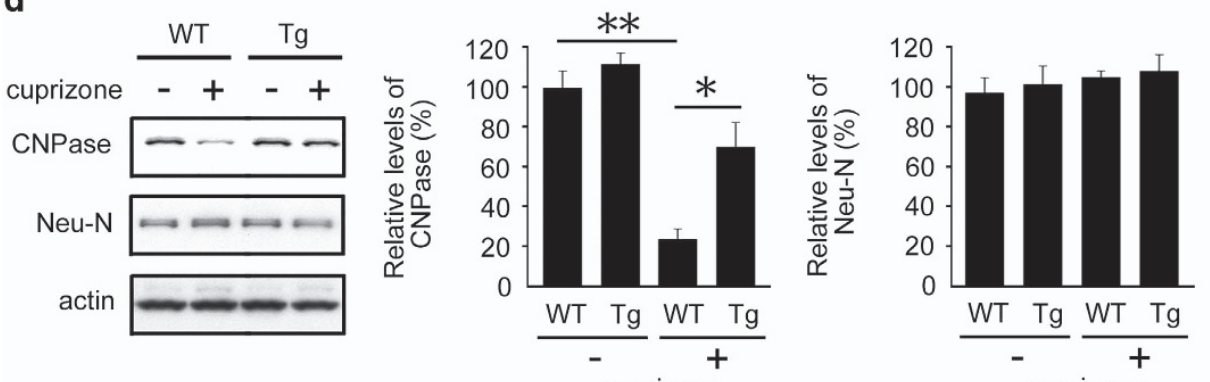

cuprizone

Figure 2 Effects of Dock3 on myelin protection in the corpus callosum of a cuprizone-induced demyelinating model. (a and b) Brain coronal sections illustrating a high intensity of LFB (a) and MBP (b) in Dock3 Tg mice compared with WT mice after cuprizone treatment for 6 weeks. Right panels are higher magnifications of the boxes shown in the left panels. Scale bars: $150 \mu \mathrm{m}$ in the left panels and $50 \mu \mathrm{m}$ in the right panels. (c) Quantitative analysis of LFB-and MBP-intensities in $\mathbf{a}$ and $\mathbf{b}$. (d) Western blot analysis displaying increased CNPase expression in the brain of Dock3 Tg mice after cuprizone treatment. The data are presented as mean \pm S.E.M. of six independent samples. The results are expressed as percentages of the normal WT mice. ${ }^{* *} P<0.01 ;{ }^{*} P<0.05$ 
Effects of cuprizone on the retina and optic nerve degeneration. As MS often induces optic neuritis that leads to visual disturbance, ${ }^{12,13}$ we next examined the effects of cuprizone on optic nerves. Immunoblot analysis of CNPase revealed small but a significant level of demyelination of optic nerves after cuprizone treatment for 12 weeks, but not 6 weeks (Figure 3a). In addition, cuprizone treatment reduced MBP-like immunoreactivity in optic nerves (Figure 3b). However, retinal morphology was normal and the degeneration of RGCs was not observed in cuprizone-treated mice (Figures $3 c$ and $d$ ). These results suggest that cuprizone induces demyelination of optic nerves, but not retinal degeneration. This model is different from experimental autoimmune encephalomyelitis (EAE), a well-studied MS model, in which RGC degeneration and optic neuritis are observed. $^{22,23}$

Effects of Dock3 on myelin protection in the optic nerves. We next examined the effects of Dock3 on the severity of demyelination in optic nerves from both the histopathological and physiological aspects. Cuprizone treatment clearly reduced MBP immunoreactivity in WT mice, but the extent of demyelination was significantly less in Dock3 Tg mice (Figures $4 a$ and b). Protection of myelin in Dock3 Tg mice was further confirmed by immunoblot analysis for CNPase expression levels (Figures $4 c$ and $d$ ). We next investigated visual functions of cuprizone-treated mice using multifocal mfERGs, an established non-invasive method for effectively measuring visual function. ${ }^{22,23}$ The response topography demonstrated that the visual function of cuprizone-treated WT mice was impaired in all visual fields, but it was clearly better preserved in cuprizone-treated Dock3 Tg mice (Figure 4e). Furthermore, quantitative analysis confirmed that the visual function in cuprizone-treated Dock3 Tg mice $\left(2.51 \pm 0.34 \mathrm{nV} / \mathrm{deg}^{2} ; n=6\right)$ was significantly better than cuprizone-treated WT mice $\left(1.67 \pm 0.26 \mathrm{nV} / \mathrm{deg}^{2} ; n=6\right)$ (Figure 4f). Taken together, these data demonstrate that Dock3 attenuates both the histological and functional aspects of cuprizone-induced demyelination in optic nerves.

Effects of Dock3 on the signal transduction in the optic nerves. We then investigated the mechanisms for attenuated optic nerve demyelination in Dock3 Tg mice. Dock3 expression in Dock3 Tg mice is about five times higher than a
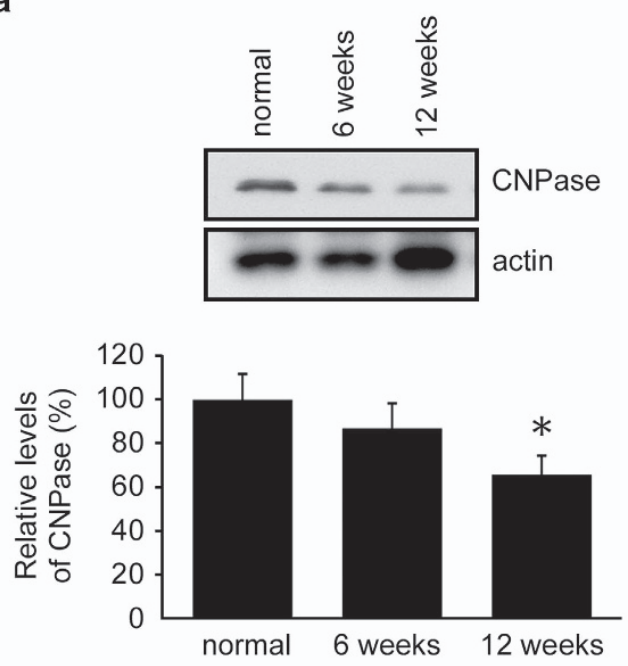

C

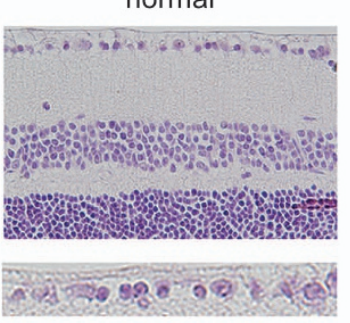

cuprizone

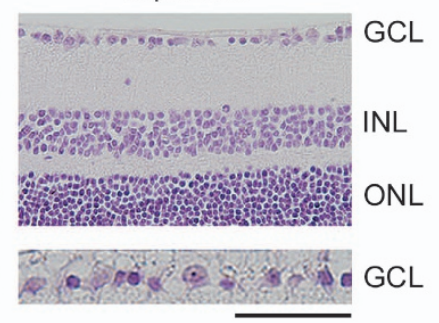

b
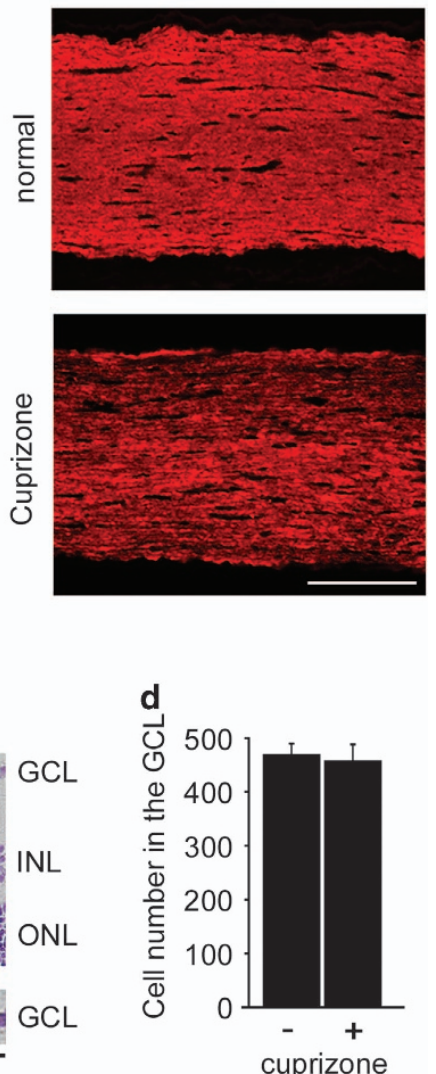

Figure 3 Effects of cuprizone on the retina and optic nerve degeneration. (a) Western blot analysis displaying decreased CNPase expression in the optic nerves after cuprizone treatment. The data are presented as mean \pm S.E.M. of six independent samples. The results are expressed as percentages of the normal WT mice. ${ }^{*} P<0.05$. (b) Optic nerve sections illustrating a decreased MBP intensity after cuprizone treatment for twelve weeks. Scale bar: $100 \mu \mathrm{m}$. (c) Hematoxylin and eosin staining of retinal sections after cuprizone treatment for twelve weeks. The microscopic image of each section within $0.5-1 \mathrm{~mm}$ from the optic disc was scanned. ganglion cell layer (GCL), ganglion cell layer; INL, inner nuclear layer; ONL, outer nuclear layer. Scale bar: $100 \mu \mathrm{m}$ and $50 \mu \mathrm{m}$ in the upper and lower panels, respectively. (d) Quantification of the retinal degeneration. The number of cells in the GCL was counted from one ora serrata through the optic nerve to the other ora serrata. The data are presented as mean \pm S.E.M. of six independent samples 
a
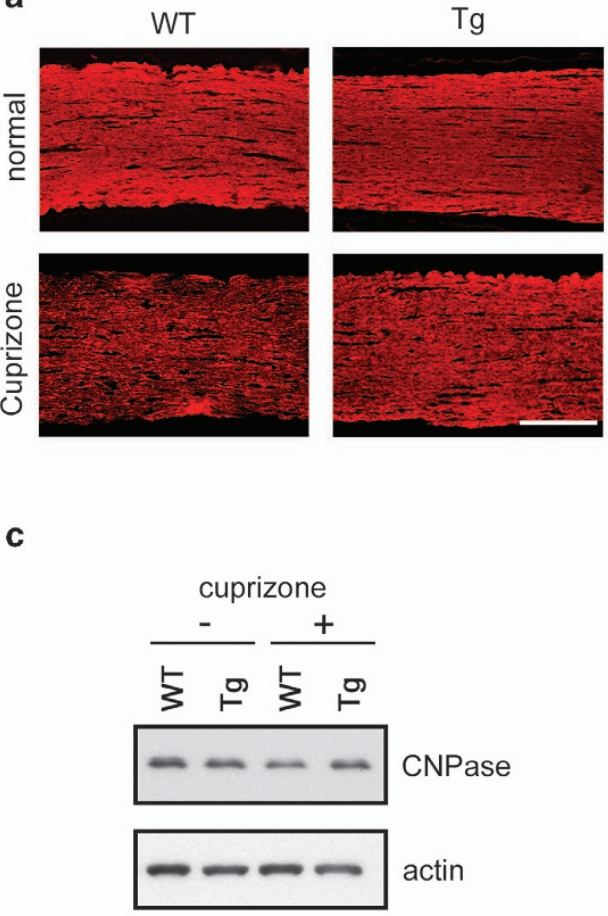

e

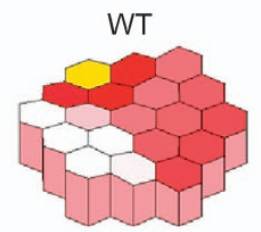

WT/ cuprizone

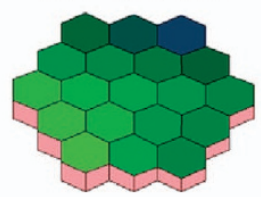

$\mathrm{Tg}$

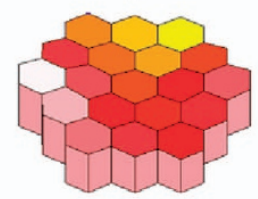

$\mathrm{Tg} /$ cuprizone

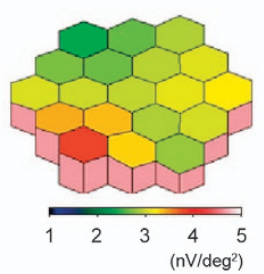

b

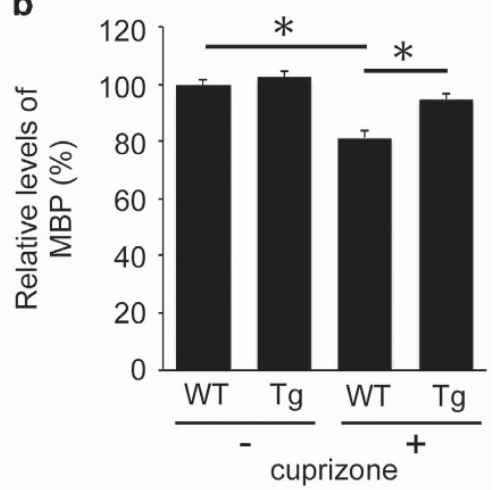

d

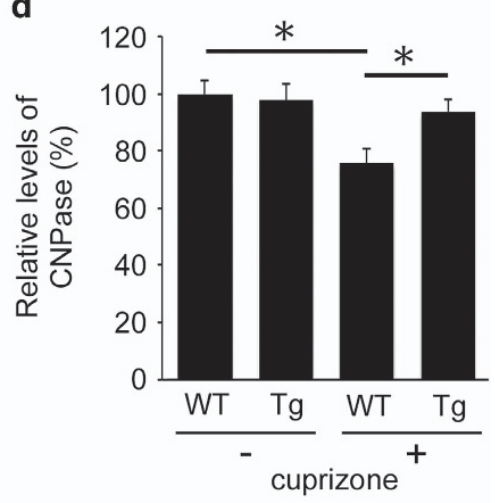

f

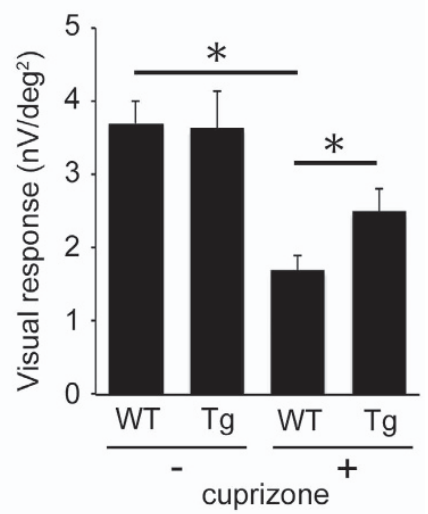

Figure 4 Effects of Dock3 on myelin protection in the optic nerves of a cuprizone-induced demyelinating model. (a) Optic nerve sections illustrating a high MBP intensity in Dock3 Tg mice compared with WT mice after cuprizone treatment for twelve weeks. Scale bar: $100 \mu \mathrm{m}$. (b) Quantitative analysis of MBP-positive areas in the optic nerves in (a). The data are presented as mean \pm S.E.M. of six independent samples. The results are expressed as percentages of the normal WT mice. ${ }^{*} P<0.05$. (c) Western blot analysis displaying increased CNPase expression in the optic nerves of Dock3 Tg mice after cuprizone treatment. (d) Quantitative analysis of CNPase expression levels in c. The data are presented as mean \pm S.E.M. of six independent samples. The results are expressed as percentages of the normal WT mice. ${ }^{*} P<0.05$. (e) The averaged visual responses from six WT and Dock3 Tg mice were examined by multifocal electroretinograms (mfERGs). The degree of retinal function is presented in the color bar. The higher score (red) indicates highly sensitive visual function and the lower score (green) indicates retinal dysfunction. Values are given in nanovolt per square degree $\left(\mathrm{nV} / \mathrm{deg}^{2}\right)$. (f) Quantitative analysis of the visual response amplitude. The sum of the response amplitudes for each stimulus element was divided by the total area of the visual stimulus. The data are presented as mean \pm S.E.M. of six independent samples. ${ }^{\star} P<0.05$

that in WT mice (Figures $5 a$ and b). ${ }^{5}$ Dock3 forms a ternary complex with engulfment and cell motility (Elmo) and RhoG, which leads to Rac1 activation and neurite outgrowth. ${ }^{24}$ Since Dock3 GEF activity is enhanced by the formation of the Dock3/Elmo complex, we next examined whether Dock3 overexpression affects Elmo expression. We found that the
Elmo expression level in Dock3 Tg mice is about three times higher than that in WT mice (Figures $5 a$ and b). Immunohistochemical analysis revealed that Elmo expression is increased in Dock3 Tg mice compared with WT mice, and Dock3 and Elmo are co-localized to CC-1-positive myelin in optic nerves (Figure 5c). 
a

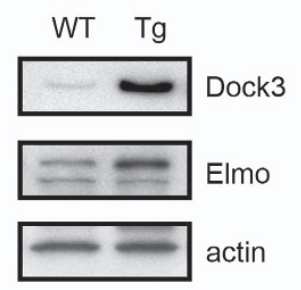

C
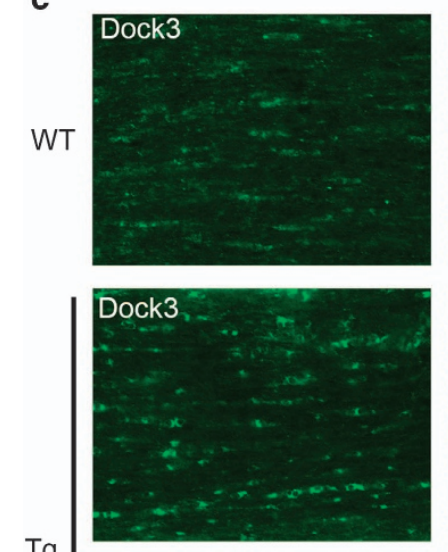

$\operatorname{Tg}$

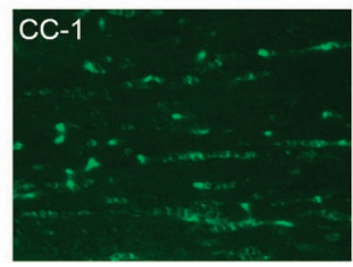

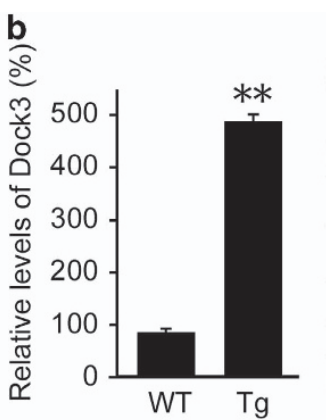

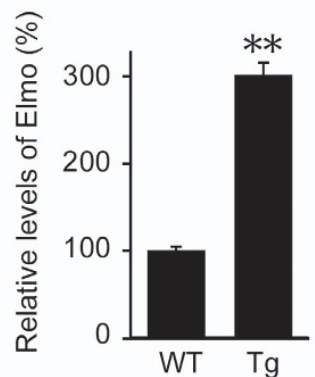

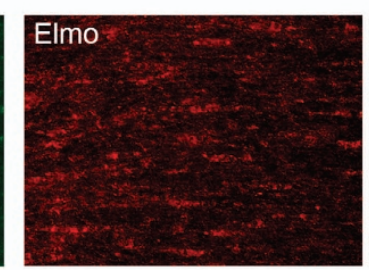
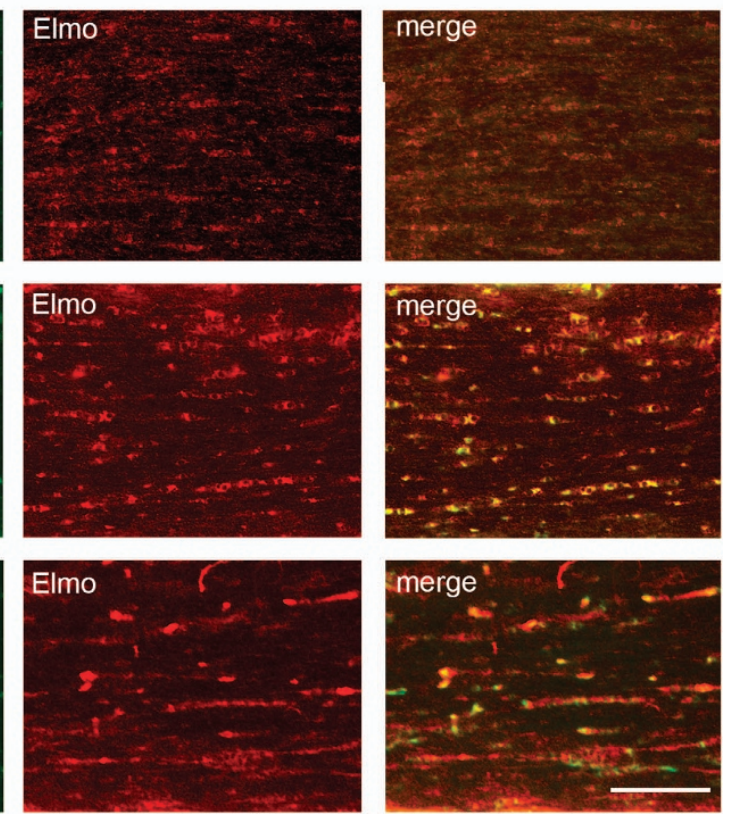

Figure 5 Effects of Dock3 on Elmo expression in the optic nerves. (a) Western blot analysis of Dock3 and Elmo in the optic nerves of WT and Dock3 Tg mice. (b) Quantitative analysis of Dock3 and Elmo proteins in WT and Dock3 Tg mice. The data are presented as mean \pm S.E.M. of six independent samples. The results are expressed as percentages of the WT mice. ${ }^{* *} P<0.01$. (c) Optic nerve sections illustrating a high intensity of Dock3 and Elmo in the optic nerve of Dock3 Tg mice compared with WT mice. Scale bar: $50 \mu \mathrm{m}$

Recent studies have shown that mitogen-activated protein kinases (MAPKs) are involved in oxidative stress-induced apoptotic cell death of oligodendrocytes. ${ }^{25,26}$ For example, ghrelin, a 28-amino acid gastric hormone, inhibits oligodendrocytes cell death in part by increasing extracellular signalregulated kinase (Erk) activation and decreasing p38 MAPK activation. ${ }^{26}$ To determine whether this is true in our model, we examined the activation of Erk, p38 MAPK and c-Jun $\mathrm{N}$-terminal kinase (JNK) in optic nerves of WT and Dock3 Tg mice, and found that only Erk activation is increased in Dock3 Tg mice (Figures 6a-c). Consistently, immunohistochemical analysis revealed that phosphorylated Erk expression in CC-1-positive myelin is increased in Dock3 Tg mice compared with WT mice (Figure 6d). These results suggest that Dock3 overexpression protects oligodendrocytes in optic nerves, at least partly, by enhancing Erk activation.

\section{Discussion}

Here we show that Dock3 is expressed in oligodendrocytes and overexpression of Dock3 ameliorated demyelination in both corpus callosum and optic nerves, and protected visual function in the cuprizone-induced demyelination model. Our findings demonstrated in vivo a novel function of a Dock family member during demyelination in the CNS. Our present results also showed that the cuprizone-induced demyelination model is useful as an animal model of optic neuritis, like EAE. ${ }^{22,23}$ We administered cuprizone for 6 weeks for the demyelination of the corpus callosum, but it required additional 6 weeks to detect demyelination of the optic nerve. This may reflect the difference in tissue sensitivity for cuprizone. For the objective analysis of the visual function in mice, we examined the second-order kernel of mfERGs, which is impaired in patients with glaucoma. ${ }^{27,28}$ To the best of our knowledge, there are no reports examining the second-order kernel in patients with optic neuritis. On the other hand, abnormalities in visual function in MS can be detected by the multifocal visual evoked potentials (MfVEP) with high sensitivity and specificity. ${ }^{29}$ Although mfVEP cannot be examined in mice, this method may be available for the early diagnosis of MS and optic neuritis. 
a

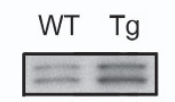

phospho Erk

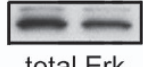

total Erk

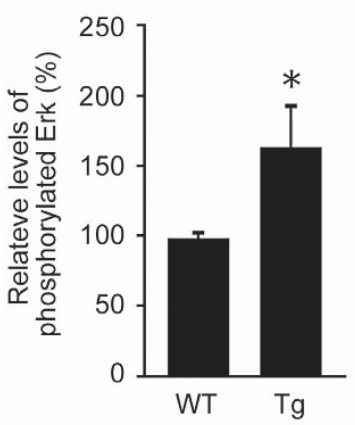

d
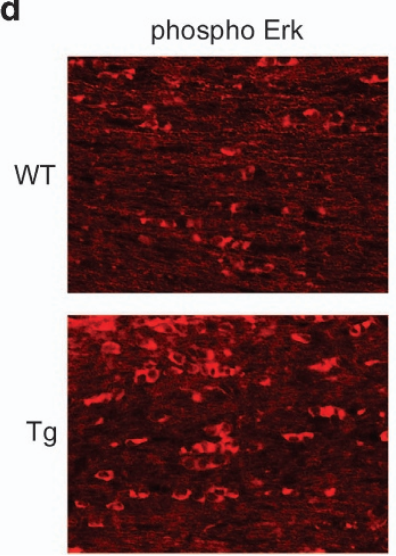

b

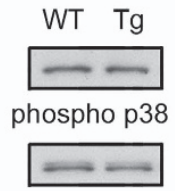

total p38

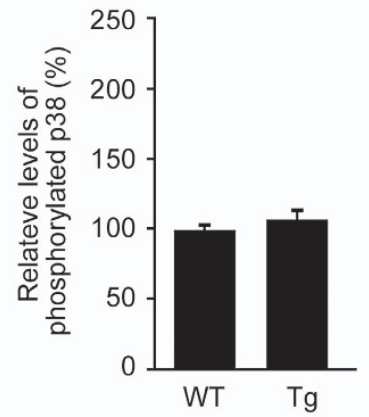

CC-1
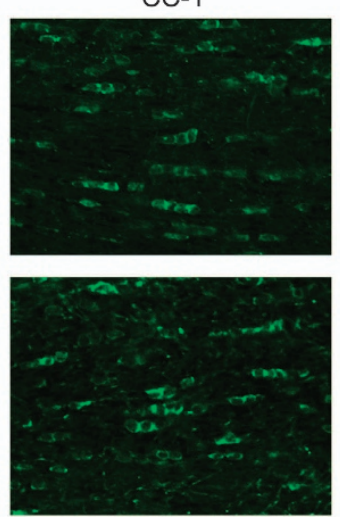
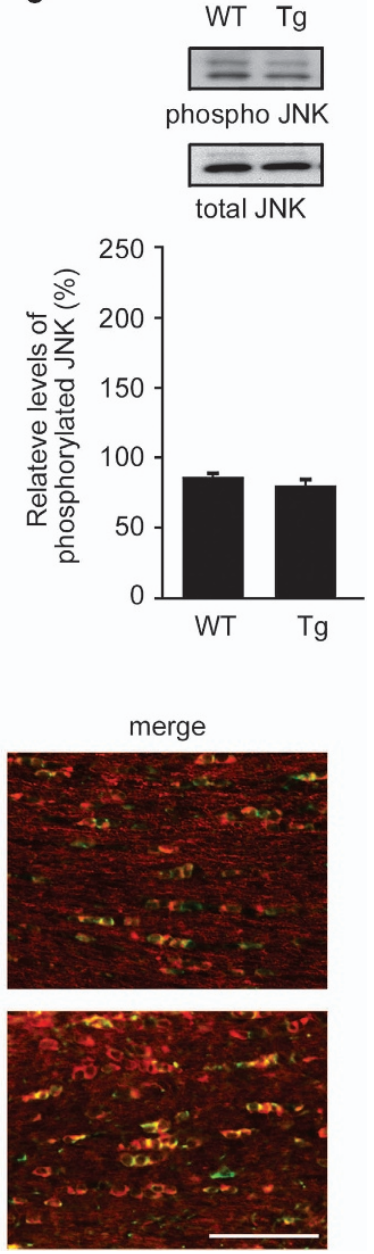

Figure 6 Effects of Dock3 on Erk activation in optic nerves. (a-c) Western blot analysis of total and phosphorylated Erk (a), p38 (b) and JNK (c) in the optic nerves of WT and Dock3 Tg mice. Relative levels of phosphorylated proteins are quantified. The data are presented as mean \pm S.E.M. of six independent samples. The results are expressed as percentages of the WT mice. ${ }^{*} P<0.05$. (d) Optic nerve sections illustrating a high intensity of phosphorylated Erk in the optic nerve of Dock3 Tg mice compared with WT mice. Scale bar: $50 \mu \mathrm{m}$

One of the underlying mechanisms for Dock3-mediated myelin protection appears to be stimulation of Erk signaling in optic nerves. Erk signaling is involved in the oligodendrocyte survival pathway after exposure to oxidative stress and Erk1 $\mathrm{KO}$ mice are known to exhibit increased susceptibility to EAE, suggesting an important role of Erk signaling during demyelination. ${ }^{26,30,31}$ It is known that Rac1 signaling is crucial to stimulate Erk signaling through Pak1-mediated control of Mek1/2 phosphorylation. ${ }^{32}$ In addition, Erk may maintain the activation of Rac1 signaling through phosphorylation and downregulation of the activity of Cdc42 GTPase-activating protein that inhibits Rac1. ${ }^{33}$ In this study, we showed that Elmo is increased in Dock3 Tg mice, suggesting more effective activation of Rac1 signaling in these mice. Taken together, Dock3 overexpression may protect myelin, at least partly, by increasing Elmo expression, enhancing Rac1 signaling, stimulating Erk signaling and driving the positive feedback loop between Rac1 and Erk. In contrast to Erk signaling, p38 signaling is known to be involved in neural cell death in the CNS. A recent study reported that inhibition of p38 stimulates survival of oligodendrocytes. ${ }^{26}$ We have previously shown that deficiency in apoptosis signal-regulating kinase 1 (ASK1), an upstream enzyme of the p38 signaling pathway, protects retinal degeneration ${ }^{34-37}$ and suppresses demyelination in EAE mice. ${ }^{22}$ Since Dock3 overexpression did not affect p38 activity in optic nerves (Figure 6b), combination of Dock3 overexpression and inhibition of ASK1-p38 signaling by specific inhibitors may be effective for the management of demyelinating diseases including MS. $22,37,38$

Gene array analyses revealed that cuprizone affects glutamate signaling during demyelination, ${ }^{39}$ suggesting that cuprizone induces glutamate neurotoxicity. We recently showed that Dock3 can ameliorate glutamate neurotoxicity by suppressing $\mathrm{N}$-methyl-D-aspartate (NMDA) receptor activities. ${ }^{4,40}$ Since these receptors are expressed in oligodendrocytes in the optic nerves, ${ }^{41}$ Dock3 may protect oligodendrocytes by suppressing cuprizone-induced glutamate neurotoxicity. Indeed, NMDA receptor blockade has been shown to be an effective treatment strategy for optic neuritis in EAE rats, ${ }^{42}$ supporting the effectiveness of Dock3mediated negative regulation of NMDA receptors and 
protection in our current model. Thus, Dock3 may provide myelin protection via multiple pathways, including activation of Erk signaling and inhibition of glutamate signaling.

It is well-known that brain-derived neurotrophic factor (BDNF) stimulation induces Erk activation in several cells and promotes cell survival. Since BDNF is decreased in the corpus callosum following cuprizone treatment, ${ }^{43}$ BDNF-Erk signaling may play an important role in demyelinating diseases. Recent studies have shown that ulinastatin, a glycoprotein which acts as a trypsin inhibitor, increased the expression of BDNF, and protected oligodendrocytes from apoptosis. ${ }^{44}$ In addition, laquinimod, an orally available synthetic compound designed for the treatment of relapsingremitting MS (RRMS), ameliorates EAE disease course via BDNF-dependent mechanisms ${ }^{45}$ and furthermore, glatiramer acetate, a medication used in RRMS, promotes oligodendrogenesis and remyelination through elevation of growth factors including BDNF. ${ }^{46}$ These accumulated evidence indicate that stimulation of BDNF signaling may be a promising therapeutic target for demyelinating disorders. Since Dock3 acts downstream of BDNF signaling, ${ }^{1,5}$ it is possible that Dock3mediated Erk activation is involved in BDNF-dependent amelioration of MS/EAE.

In this study, we have shown the importance of Dock3 expression in oligodendrocytes during demyelinating environment. However, we cannot exclude the possibility that Dock3 expression in cells other than oligodendrocytes may play important roles for the ameliorated demyelination in Dock3 Tg mice. For example, microglia that invade the brain and optic nerves during demyelination may release several trophic factors including BDNF, which stimulate oligodendrocyte protection. ${ }^{47,48}$ On the other hand, stimulation of TrkB on astrocytes may drive nitric oxide production and neurodegeneration in EAE. ${ }^{49}$ Thus, further studies are required to determine the cell type specific functions of Dock3 and BDNF in vivo. In conclusion, our findings raise intriguing possibilities for the management of demyelinating diseases by Dock3 overexpression in combination with agents that increase trophic factor release. To this end, we are currently investigating therapeutic application of Dock3 using Dock3 virus vectors in disease models.

\section{Materials and Methods \\ Mice. Experiments were performed using Dock3 $\mathrm{Tg}_{\text {mice }}{ }^{4-6}$ in accordance with the Tokyo Metropolitan Institute of Medical Science Guidelines for the Care and Use of Animals. Dock3 Tg mice overexpressing WT Dock3 were generated under the control of an actin promoter. Dock3 Tg mice showed increased expression levels of Dock3 in many tissues, especially in the optic nerve ( $\sim 5.6$-fold) and retina ( $\sim 2.3$-fold), but the development and structure of such tissues were normal. ${ }^{5}$ C57BL/6J mice were obtained from CLEA Japan (Tokyo, Japan).}

Culture cells from brain. Primary cultured hippocampal neurons were prepared from E16 mice. ${ }^{5}$ Primary astrocytes were obtained as previously reported. ${ }^{22}$ Primary oligodendrocytes were obtained from mixed glial cultures as previously described. ${ }^{50}$ Cerebral cortices from 1-3-day-old mice were excised, meninges removed and cortices minced into small pieces. Subsequently, the tissue was dissociated by incubation with $0.25 \%$ trypsin at $37^{\circ} \mathrm{C}$ for $15 \mathrm{~min}$, and the resultant tissue suspension was triturated to yield a single cell suspension. The cells were seeded on a $75 \mathrm{~cm}^{2}$ tissue culture flask in Dulbecco's Modified Eagle Medium (DMEM) containing 10\% fetal bovine serum. Complete confluence was reached in $7-10$ days at $37^{\circ} \mathrm{C} / 5 \% \mathrm{CO}_{2}$. The culture flasks were shaken for 16-20 h (150 r.p.m. at $37^{\circ} \mathrm{C}$ ) in a temperature-controlled shaker to separate the oligodendrocyte precursor cells (OPCs) grown on the top of the confluent layer of astrocytes. Purified OPCs were cultured on a poly-D-lysine-coated dish in DMEM supplemented with the following reagents (Sigma, St. Louis, MO, USA; unless otherwise stated): $5 \mathrm{~g} / \mathrm{ml}$ bovine insulin, $50 \mathrm{~g} / \mathrm{ml}$ human transferrin, $100 \mathrm{~g} / \mathrm{ml}$ bovine serum albumin fraction V, $66 \mathrm{ng} / \mathrm{ml}$ progesterone, $16 \mathrm{ng} / \mathrm{ml}$ putrescine, $5 \mathrm{ng} / \mathrm{ml}$ sodium selenite, $400 \mathrm{ng} / \mathrm{ml}$ thyroxine, $400 \mathrm{ng} / \mathrm{ml}$ tri-iodothyroxine (Biofluids Inc., Rockville, MD, USA), $10 \mathrm{ng} / \mathrm{ml}$ PDGF-AA and $10 \mathrm{ng} / \mathrm{ml} \mathrm{bFGF.} \mathrm{To} \mathrm{obtain}$ differentiated mature oligodendrocytes, PDGF-AA was removed.

Cuprizone-induced demyelination. WT and Dock3 Tg mice were fed with $0.2 \%$ cuprizone (bis-cyclohexanone oxaldihydrazone; Sigma) mixed into a ground standard rodent chow to induce demyelination. Cuprizone feeding was maintained for 6 or 12 weeks for corpus callosum or optic nerves, respectively. Mice were killed after the treatment period and samples were subjected to histological and immunoblot analysis.

Histopathology and immunohistochemistry. Optic nerves and corpus callosum were examined as previously reported. ${ }^{22,23}$ Paraffin embedded sections of $7 \mu \mathrm{m}$ thickness were cut and stained with hematoxylin and eosin or LFB. For quantitative analysis, the number of neurons in the ganglion cell layer was counted from one ora serrata through the optic nerve to the other ora serrata. For the detection of Dock3 in oligodendrocytes in the optic nerve, fresh tissues were removed and frozen on dry ice. Serial cross-sections $(10 \mu \mathrm{m})$ were prepared using a cryostat, and immersed in $4 \%$ paraformaldehyde for $1 \mathrm{~h}$ at $4{ }^{\circ} \mathrm{C}$ before staining. Immunohistochemistry was performed using the following primary antibodies: Dock3 (1:200), ${ }^{4}$ Elmo (1:200, Everest Biotech, Oxfordshire, UK), MBP (1:2000; Santa Cruz, Santa Cruz, CA, USA), CC-1 (1:100; Calbiochem, Gibbstown, NJ, USA) and phosphorylated Erk (1:200; BD biosciences, San Diego, CA, USA). Quantitative analysis of the stained region was carried out using ImageJ (NIH, Bethesda, MD, USA).

Immunoblot analysis. Immunoblotting was carried out as previously reported. ${ }^{4}$ Brains, optic nerves and cultured oligodendrocytes were freshly isolated, and then homogenized. Samples were separated on a sodium dodecyl sulfate-PAGE and subsequently transferred to an Immobilon-P filter (Millipore, Billerica, MA, USA). Membranes were incubated with an antibody against Dock3 (1:1000), Neu-N (1:1000, Millipore), NG2 $(1: 500$, Millipore), GFAP $(1: 500$, Santa Cruz), CNPase (1:1000, Sigma), Elmo, total Erk, phosphorylated Erk, total p38, phosphorylated p38, total JNK, phosphorylated JNK or actin (1:1000; BD Biosciences).

mfERG. Mice at 6 and 18 weeks were anesthetized by intraperitoneal injection of sodium pentobarbital. The pupils were dilated with $0.5 \%$ phenylephrine hydrochloride and $0.5 \%$ tropicamide. mfERGs were recorded using a VERIS 6.0 system (Electro-Diagnostic Imaging, Redwood City, CA, USA). The visual stimulus consisted of seven hexagonal areas scaled with eccentricity. The stimulus array was displayed on a high-resolution black and white monitor driven at a frame rate of $100 \mathrm{~Hz}$. The second-order kernel, which is impaired in patients with glaucoma, was analyzed. ${ }^{22,23}$

Statistics. For statistical comparison of two samples, we used a two-tailed Student's $t$-test. Data are presented as mean \pm S.E.M. $P<0.05$ was regarded as statistically significant.

\section{Conflict of Interest}

The authors declare no conflict of interest.

Acknowledgements. This study was supported by the Ministry of Education, Culture, Sports, Science and Technology of Japan (KN, AK, CH, TH) and the Funding Program for Next Generation World-Leading Researchers (NEXT Program; TH).

1. Namekata K, Kimura A, Kawamura K, Harada C, Harada T. Dock GEFs and their therapeutic potential: Neuroprotection and axon regeneration. Prog Retin Eye Res 2014; e-pub ahead of print 11 July 2014; doi:10.1016/j.preteyeres.2014.06.005.

2. Namekata K, Enokido Y, Iwasawa K, Kimura H. MOCA induces membrane spreading by activating Rac1. J Biol Chem 2004; 279: 14331-14337. 
3. Laurin M, Côté JF. Insights into the biological functions of Dock family guanine nucleotide exchange factors. Genes Dev 2014; 28: 533-547.

4. Namekata K, Kimura A, Kawamura K, Guo X, Harada C, Tanaka K et al. Dock3 attenuates neural cell death due to NMDA neurotoxicity and oxidative stress in a mouse model of normal tension glaucoma. Cell Death Differ 2013; 20: 1250-1256.

5. Namekata K, Harada C, Taya C, Guo X, Kimura H, Parada LF et al. Dock3 induces axonal outgrowth by stimulating membrane recruitment of the WAVE complex. Proc Natl Acad Sci USA 2010; 107: 7586-7591.

6. Namekata K, Harada C, Guo X, Kimura A, Kittaka D, Watanabe H et al. Dock3 stimulates axonal outgrowth via GSK-3 $\beta$-mediated microtubule assembly. J Neurosci 2012; 32 264-274.

7. Kashiwa A, Yoshida $\mathrm{H}$, Lee $\mathrm{S}$, Paladino $\mathrm{T}$, Liu $\mathrm{Y}$, Chen $\mathrm{Q}$ et al. Isolation and characterization of novel presenilin binding protein. J Neurochem 2000; 75: 109-116.

8. Bertram L, Lill CM, Tanzi RE. The genetics of Alzheimer disease: back to the future Neuron 2010; 68: 270-281.

9. Guo L, Salt TE, Luong V, Wood N, Cheung W, Maass A et al. Targeting amyloid- $\beta$ in glaucoma treatment. Proc Natl Acad Sci USA 2007; 104: 13444-13449.

10. Tamura H, Kawakami H, Kanamoto T, Kato T, Yokoyama T, Sasaki K et al. High frequency of open-angle glaucoma in Japanese patients with Alzheimer's disease. J Neurol Sci 2006; 246: 79-83.

11. de Silva MG, Elliott K, Dahl HH, Fitzpatrick E, Wilcox S, Delatycki M et al. Disruption of a novel member of a sodium/hydrogen exchanger family and DOCK3 is associated with an attention deficit hyperactivity disorder-like phenotype. J Med Genet 2003; 40: 733-740.

12. Jarius S, Paul F, Franciotta D, Waters P, Zipp F, Hohlfeld R et al. Mechanisms of disease: aquaporin-4 antibodies in neuromyelitis optica. Nat Clin Pract Neurol 2008; 4 : 202-214.

13. Matiello M, Lennon VA, Jacob A, Pittock SJ, Lucchinetti CF, Wingerchuk DM et al NMO-IgG predicts the outcome of recurrent optic neuritis. Neurology 2008; 70: 2197-2200.

14. Benjamins JA Nedelkoska L, Bealmear B, Lisak RP. ACTH protects mature oligodendroglia from excitotoxic and inflammation-related damage in vitro. Glia 2013; 61: $1206-1217$.

15. Matute $C$, Alberdi E, Domercq M, Pérez-Cerdá F, Pérez-Samartín A, Sánchez-Gómez MV The link between excitotoxic oligodendroglial death and demyelinating diseases. Trends Neurosci 2001; 24: 224-230.

16. Lipton SA. NMDA receptors, glial cells, and clinical medicine. Neuron 2006; 50: 9-11.

17. Matute C. Oligodendrocyte NMDA receptors: a novel therapeutic target. Trends Mol Med 2006; 12: 289-292.

18. Matsushima GK, Morell P. The neurotoxicant, cuprizone, as a model to study demyelination and remyelination in the central nervous system. Brain Pathol 2001; 11 107-116.

19. Mason JL, Toews A, Hostettler JD, Morell P, Suzuki K, Goldman JE et al. Oligodendrocytes and progenitors become progressively depleted within chronically demyelinated lesions. Am J Pathol 2004; 164: 1673-1682.

20. Kipp M, Clarner T, Dang J, Copray S, Beyer C. The cuprizone animal model: new insights into an old story. Acta Neuropathol 2009; 118: 723-736.

21. Mueller AM, Nassery A, Conlon H, Liu X, Jun E, Yoon BH et al. Effects of intraventricular methotrexate administration on Cuprizone-induced demyelination in mice. Front $\mathrm{Mol}$ Neurosci 2013; 6: 34

22. Guo X, Harada C, Namekata K, Matsuzawa A, Camps M, Ji H et al. Regulation of the severity of neuroinflammation and demyelination by TLR-ASK1-p38 pathway. EMBO Mol Med 2010; 2: 504-515.

23. Guo X, Harada C, Namekata K, Mitamura Y, Yoshida H, Matsumoto Y et al. Delayed onse of experimental autoimmune encephalomyelitis in Olig1 deficient mice. PLoS One 2010; 5 : e13083.

24. Namekata K, Watanabe H, Guo X, Kittaka D, Kawamura K, Kimura A et al. Dock3 regulates BDNF-TrkB signaling for neurite outgrowth by forming a ternary complex with Elmo and RhoG. Genes Cells 2012; 17: 688-697.

25. Fragoso G, Martínez-Bermúdez AK, Liu HN, Khorchid A, Chemtob S, Mushynski WE et al. Developmental differences in $\mathrm{H}_{2} \mathrm{O}_{2}$-induced oligodendrocyte cell death: role of glutathione, mitogen-activated protein kinases and caspase 3. J Neurochem 2004; 90 392-404

26. Lee JY, Oh TH, Yune TY. Ghrelin inhibits hydrogen peroxide-induced apoptotic cell death of oligodendrocytes via ERK and p38MAPK signaling. Endocrinology 2011; 152 2377-2386

27. Sutter EE, Bearse MA Jr. The optic nerve head component of the human ERG. Vision Res 1999; 39: 419-436.

28. Harada T, Harada C, Nakamura K, Quah HA, Okumura A, Namekata K et al. The potential role of glutamate transporters in the pathogenesis of normal tension glaucoma. $J$ Clin Invest 2007; 117: 1763-1770.

29. Laron M, Cheng H, Zhang B, Schiffman JS, Tang RA, Frishman LJ. Assessing visual pathway function in multiple sclerosis patients with multifocal visual evoked potentials. Mult Scler 2009; 15: 1431-1441.
30. Mronga T, Stahnke T, Goldbaum O, Richter-Landsberg C. Mitochondrial pathway is involved in hydrogen-peroxide-induced apoptotic cell death of oligodendrocytes. Glia 2004; 46: 446-455.

31. Agrawal A, Dillon S, Denning TL, Pulendran B. ERK1\%- mice exhibit Th1 cell polarization and increased susceptibility to experimental autoimmune encephalomyelitis. J Immunol 2006; 176: 5788-5796.

32. Wang Z, Pedersen E, Basse A, Lefever T, Peyrollier K, Kapoor S et al. Rac1 is crucial for Ras-dependent skin tumor formation by controlling Pak1-Mek-Erk hyperactivation and hyperproliferation in vivo. Oncogene 2010; 29: 3362-3373.

33. Tcherkezian J, Danek El, Jenna S, Triki I, Lamarche-Vane N. Extracellular signal-regulated kinase 1 interacts with and phosphorylates CdGAP at an important regulatory site. Mol Cell Biol 2005; 25: 6314-6329.

34. Harada C, Nakamura K, Namekata K, Okumura A, Mitamura Y, lizuka Y et al. Role of apoptosis signal-regulating kinase 1 in stress-induced neural cell apoptosis in vivo. Am J Pathol 2006; 168: 261-269.

35. Harada C, Namekata K, Guo X, Yoshida H, Mitamura Y, Matsumoto Y et al. ASK1 deficiency attenuates neural cell death in GLAST-deficient mice, a model of normal tension glaucoma. Cell Death Differ 2010; 17: 1751-1759.

36. Hattori K, Naguro I, Runchel C, Ichijo $\mathrm{H}$. The roles of ASK family proteins in stress responses and diseases. Cell Commun Signal 2009; 7: 9.

37. Katome T, Namekata K, Guo X, Semba K, Kittaka D, Kawamura K et al. Inhibition of ASK1p38 pathway prevents neural cell death following optic nerve injury. Cell Death Differ 2013; 20: $270-280$.

38. Krementsov DN, Thornton TM, Teuscher C, Rincon M. The emerging role of p38 mitogenactivated protein kinase in multiple sclerosis and its models. Mol Cell Biol 2013; 33: 3728-3734

39. Azami Tameh A, Clarner T, Beyer C, Atlasi MA, Hassanzadeh G, Naderian H. Regional regulation of glutamate signaling during cuprizone-induced demyelination in the brain. Ann Anat 2013; 195: 415-423.

40. Bai N, Hayashi H, Aida T, Namekata K, Harada T, Mishina M et al. Dock3 interaction with a glutamate-receptor NR2D subunit protects neurons from excitotoxicity. Mol Brain 2013; 6: 22

41. Salter MG, Fern R. NMDA receptors are expressed in developing oligodendrocyte processes and mediate injury. Nature 2005; 438: 1167-1171.

42. Sühs KW, Fairless R, Williams SK, Heine K, Cavalié A, Diem R. N-methyl-D-aspartate receptor blockade is neuroprotective in experimental autoimmune optic neuritis. $J$ Neuropathol Exp Neurol 2014; 73: 507-518.

43. Fulmer CG, VonDran MW, Stillman AA, Huang Y, Hempstead BL, Dreyfus CF. Astrocyte-derived BDNF supports myelin protein synthesis after cuprizone-induced demyelination. J Neurosci 2014; 34: 8186-8196.

44. Shu Y, Yang Y, Qiu W, Lu Z, Li Y, Bao J et al. Neuroprotection by ulinastatin in experimental autoimmune encephalomyelitis. Neurochem Res 2011; 36: 1969-1977.

45. Thöne J, Ellrichmann G, Seubert S, Peruga I, Lee DH, Conrad R et al. Modulation of autoimmune demyelination by laquinimod via induction of brain-derived neurotrophic factor. Am J Pathol 2012; 180: 267-274.

46. Skihar V, Silva C, Chojnacki A, Döring A, Stallcup WB, Weiss S et al. Promoting oligodendrogenesis and myelin repair using the multiple sclerosis medication glatiramer acetate. Proc Natl Acad Sci USA 2009; 106: 17992-17997.

47. Harada T, Harada C, Kohsaka S, Wada E, Yoshida K, Ohno S et al. Microglia-Müller glia cell interactions control neurotrophic factor production during light-induced retinal degeneration. J Neurosci 2002; 22: 9228-9236.

48. Mitew S, Hay CM, Peckham H, Xiao J, Koenning M, Emery B. Mechanisms regulating the development of oligodendrocytes and central nervous system myelin. Neuroscience 2013; S0306-4522: 00976-00977.

49. Colombo E, Cordiglieri C, Melli G, Newcombe J, Krumbholz M, Parada LF et al. Stimulation of the neurotrophin receptor TrkB on astrocytes drives nitric oxide production and neurodegeneration. J Exp Med 2012; 209: 521-535.

50. Chen Y, Balasubramaniyan V, Peng J, Hurlock EC, Tallquist M, Li J et al. Isolation and culture of rat and mouse oligodendrocyte precursor cells. Nat Protoc 2007; 2: 1044-1051.

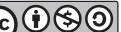

Cell Death and Disease is an open-access journal published by Nature Publishing Group. This work is licensed under a Creative Commons Attribution-NonCommercialShareAlike 3.0 Unported License. The images or other third party material in this article are included in the article's Creative Commons license, unless indicated otherwise in the credit line; if the material is not included under the Creative Commons license, users will need to obtain permission from the license holder to reproduce the material. To view a copy of this license, visit http://creativecommons.org/licenses/ by-nc-sa/3.0/ 